\title{
BMJ Open Abdominal aortic calcification, bone mineral density and fractures: a systematic review and meta- analysis protocol
}

\author{
Alexander J Rodríguez, ${ }^{1}$ Kevin Leow, ${ }^{2}$ Pawel Szulc, ${ }^{3}$ David Scott, ${ }^{1}$ Peter Ebeling, ${ }^{1}$ \\ Marc Sim, ${ }^{\circ, 5}$ Germaine Wong, ${ }^{2}$ Wai H Lim, ${ }^{6,7}$ John T Schousboe, ${ }^{8}$ Douglas P Kiel, ${ }^{9}$ \\ Richard L Prince, ${ }^{7,10}$ Joshua R Lewis ${ }^{2,4,5}$
}

To cite: Rodríguez AJ, Leow K, Szulc $P$, et al. Abdominal aortic calcification, bone mineral density and fractures: a systematic review and metaanalysis protocol. BMJ Open 2019;9:e026232. doi:10.1136/ bmjopen-2018-026232

- Prepublication history and additional material for this paper are available online. To view these files, please visit the journal online (http://dx.doi org/10.1136/bmjopen-2018026232).

Received 23 August 2018 Revised 19 December 2018 Accepted 31 January 2019
Check for updates

(C) Author(s) (or their employer(s)) 2019. Re-use permitted under CC BY-NC. No commercial re-use. See rights and permissions. Published by BMJ.

For numbered affiliations see end of article.

Correspondence to Dr Joshua R Lewis; joshua.lewis@uwa.edu.au

\section{ABSTRACT}

Introduction Abdominal aortic calcification (AAC) is associated with low bone mass and increased fracture risk. Two previous meta-analyses have investigated the association between AAC and fracture. However, these meta-analyses only identified articles until December 2016, undertook limited searches and did not explore potential sources of between-study heterogeneity. We aim to undertake a sensitive and comprehensive assessment of the relationship between $\mathrm{AAC}$, bone mineral density (BMD) as well as prevalent and incident fractures.

Methods We will search MEDLINE, EMBASE, Web of Science core collection and Google Scholar (top 200 articles sorted by relevance) from their inception to 1 June 2018. Reference lists of included studies and previous systematic reviews will be hand searched for additional eligible studies. Retrospective and prospective cohort studies (crosssectional, case-control and longitudinal) reporting the association between $\mathrm{AAC}, \mathrm{BMD}$ and fracture at any site will be included. At least two investigators will independently: (A) evaluate study eligibility and extract data, with a third investigator to adjudicate when discrepancies occur, (B) assess study quality by the Newcastle-0ttawa Scale for each cohort/study. The meta-analysis will be reported in adherence to the Meta-analysis of Observational Studies in Epidemiology criteria. AAC will be grouped as either: (1) AAC present or absent, (2) AAC categorised as 'low' (referentlowest reported group) versus 'high' (all other groups) or (3) dose-response when AAC was assessed in $\geq 3$ groups. Where primary event data were reported in individual studies, pooled risk differences and risk ratios with $95 \% \mathrm{Cl}$ will be calculated, from which, a summary estimate will be determined using DerSimonian-Laird random effects models. For the AAC and BMD pooled analyses, estimates will be expressed as standardised mean difference with $95 \% \mathrm{Cl}$. We will examine the likelihood of publication bias and where possible, investigate potential reasons for between-study heterogeneity using subgroup analyses and meta-regression.

Ethics and dissemination The study will be submitted to a peer- reviewed journal and disseminated via research presentations.

PROSPERO registration number CRD42018088019.
Strengths and limitations of this study

- This study will use meta-regression to identify sources of heterogeneity and identify subgroups or subpopulations where abdominal aortic calcification (AAC) is more or less predictive of poorer outcomes.

- To our knowledge, there has been no systematic review and meta-analysis that has investigated the association between AAC and bone mineral density (BMD), which is along the hypothesised causal pathway to fracture.

- The main limitation of this review is that causality cannot be established due to the observational nature of the studies.

- A further limitation is the differences in imaging modality, measurement and reporting of AAC across studies but we attempted to overcome this by exploring these aspects in prespecified subanalyses.

\section{INTRODUCTION}

Vascular and bone diseases are both chronic age-related disease that share many common dietary and lifestyle risk factors and cause considerable morbidity and mortality. ${ }^{1}$ Atherosclerotic lesions in the abdominal aorta generally begin around the major vessel bifurcations and branching arteries such as the inferior mesenteric artery and the lumbar arteries that supply blood and nutrients to the lumbar vertebrae. ${ }^{2}$ Occlusion of these vessels may causes ischaemia in the lumbar spine and may result in disc degeneration and asymptomatic vertebral fractures. ${ }^{3}$ Additionally, the underlying processes regulating arterial calcification share many similarities to bone physiology and calcified atherosclerotic plaques release both local and systemic osteochondrogenic factors that may affect regional and systemic bone homoeostasis. ${ }^{5}$ Conversely circulating levels of factors regulating bone homoeostasis may also regulate vascular 
calcifications ${ }^{6}$ with a number of studies demonstrating osteoporosis and bone mineral density (BMD) being a risk factor for cardiovascular disease $(\mathrm{CVD}) .^{78}$

Assessment of lateral spine images is often undertaken to detect prevalent vertebral fractures and has been shown to improve fracture prediction. ${ }^{9-11}$ These images can also be used to assess the degree of abdominal aortic calcification (AAC). To date, there are conflicting findings as to whether AAC is associated with BMD and fractures and whether or not these associations are due to ageing, shared fracture risk factors or are a non-traditional independent fracture risk factor. Recent meta-analyses published in $2016{ }^{12}$ and $2017,{ }^{13}$ looking at observational studies, showed that people with any or high AAC were at greater risk of fractures than those with no or low AAC. However, the previous studies by $\mathrm{Chen}$ and $\mathrm{Yu}^{12}$ and Wei $e t a l^{13}$ only identified a limited number of articles due to the search strategies employed (searches found 91 and 105 articles, respectively) and the meta-analyses missed many of the known studies in the area (by way of example-both studies missed Wang et al. ${ }^{14}$ ). For example, our recent search identified 1561 potentially eligible reports. Furthermore, both meta-analyses identified unexplained moderate-high heterogeneity that needs to be explored further. As such uncertainty exists as to the importance of identifying AAC for incident fracture risk, particularly with respect to AAC cut-points, types of fracture and potential explanations for the observed between-study heterogeneity. We will, therefore, undertake a meta-analysis of studies reporting on AAC, BMD at any site and prevalent and incident fractures at any site.

\section{OBJECTIVES}

1. To determine the association between AAC with BMD at any site.

2. To determine the association between AAC with prevalent fractures (cross-sectional) by reported prevalent fracture sites.

3. To determine the association between AAC with incident fractures by reported incident fracture sites.

4. To assess the impact of potential effect modifiers, including aspects of clinical, methodological and statistical heterogeneity on previous published findings.

\section{METHODS AND ANALYSIS}

The systematic review and meta-analysis reported in adherence to the Meta-analysis of Observational Studies in Epidemiology reporting criteria. ${ }^{15}$

\section{Patient and public involvement statement}

There is no patient or public involved in this systematic review/meta-analysis.

\section{Eligibility criteria for studies included in this review} Criteria for considering studies for review

a. Observational studies in humans. These include cohort (both retrospective and prospective cohort studies), case-control and cross-sectional studies that report eligible exposure(s) and outcome(s).

b. AAC assessed by any methodology.

c. Report any BMD measure or prevalent or incident fracture outcome.

\section{Exclusion criteria}

a. Reviews of existing literature.

\section{Exposure}

AAC identified from either radiography, dual X-ray absorptiometry (DXA) machine or CT. AAC will be presented as:

a. AAC present or absent.

b. AAC categorised as low (referent-lowest reported group) versus moderate to high (all other reported groups combined).

c. AAC dose-response when AAC was assessed in three or more groups categorised as low (lowest reported category), moderate (middle reported category[ies]) and high (highest reported category).

\section{Outcomes}

1. BMD (by site).

2. Prevalent fractures (by fracture site).

3. Incident fractures (by fracture site).

Cohort characteristics for meta-regression (where available)

- Cohort age (cohort mean).

- Gender (\% female).

- Years since menopause (cohort mean).

- Hormone replacement therapy (\%).

- Modality of assessing AAC (DXA, standard radiograph or CT).

- Cut-points chosen for comparison (low vs high, tertiles etc).

- Diabetes (\% of cohort).

- Current smoker (\% of cohort).

- History of smoking (\% of cohort).

- Body mass index (cohort mean).

- Chronic kidney disease (\% of cohort).

- History of CVD (\% of cohort).

- Location of study (Europe, Asia-Pacific, North America), that is, are association consistent across ethnicities and nation wealth.

- Prevalence of CVD medication use (\% of cohort).

- History of fracture (\% of cohort).

\section{Study design}

Search strategies

A comprehensive literature search within MEDLINE, Web of Science core collection and EMBASE databases will be conducted to source all possibly relevant studies for review, without language restriction. Google Scholar will be searched for the top 200 articles sorted by relevance. The search terms will be combined with the boolean 'AND' to find all potentially relevant studies. Conference proceedings and abstracts will also be evaluated. A hand 
Table 1 Example search strategies

\begin{tabular}{|c|c|c|}
\hline Keyword & MEDLINE & Embase \\
\hline Population=adults & No search strategy & No search strategy \\
\hline $\begin{array}{l}\text { Intervention/Test=aortic } \\
\text { calcification }\end{array}$ & $\begin{array}{l}\text { exp Vascular Calcification/orexp Calcinosis/ } \\
\text { orexp Vascular Diseases/orarterial calcification. } \\
\text { mp or exp Arteriosclerosis/orexp Arterial } \\
\text { Occlusive Diseases/orexp Aortic Diseases/ } \\
\text { or aortic.mp or vascular calcifications.mp. } \\
\text { or exp Vascular Calcification/or calcified } \\
\text { atherosclerosis.mp or calcification.mp or } \\
\text { calcified atherosclerotic plaque.mp or arterial } \\
\text { calcium.mp or aortic calcification.mp or aorta } \\
\text { calcification.mp and aort\$.mp and calc\$.mp }\end{array}$ & $\begin{array}{l}\text { vascular calcification.mp. or exp blood vessel } \\
\text { calcification/orartery calcification.mp. or exp } \\
\text { artery calcification/or exp coronary artery } \\
\text { disease/or exp arteriosclerosis/or calcified } \\
\text { atherosclerosis.mp or arterial calcium. } \\
\mathrm{mp} \text { or calcified atherosclerotic plaque.mp } \\
\text { or calcification.mp or aortic calcification. } \\
\mathrm{mp} \text { or aorta calcification.mp or vascular } \\
\text { calcifications.mp or arteriosclerosis.mp or } \\
\text { extracoronary.mp and aort\$.mp and calc\$.mp }\end{array}$ \\
\hline Methodology=observational & No search strategy & No search strategy \\
\hline Comparator=None & No search strategy & No search strategy \\
\hline
\end{tabular}

The reference lists of recent literature reviews and guidelines will be hand searched for possibly relevant studies.

search of reference lists of eligible studies and previous meta-analyses will also be undertaken. Non-English papers will be translated and evaluated for eligibility. If more than one publication of a study is retrieved, articles with the most up to date and complete information will be included, although additional unique data from all sources will be considered and included when relevant. Examples of the search strategy are shown in table 1.

\section{Process for selecting studies}

Two or more independent authors (AR, KL, MS and JRL) will assess retrieved citations to assess studies for eligibility. Briefly, the process for selecting studies for inclusion in the review and meta-analysis will be as follows: merge all identified records using EndNote; remove duplicate records of the same report; retrieve full text of the potentially relevant reports; link together multiple reports of the same study (using the first or largest report as the primary record and subsequent reports to supplement other data); examine full-text reports for compliance with eligibility criteria; correspond with investigators, where appropriate, to clarify study eligibility and request missing data; make final decisions on study inclusion. Discrepancies about inclusion will be resolved via iteration and consensus or a third reviewer if consensus cannot be reached between the two reviewers. Excluded studies identified that may plausibly be expected to be an included will be reported in online supplementary data with a detailed explanation for the reason of exclusion.

\section{Risk of bias and quality assessment}

The risk of bias for observational studies will be assessed using the Newcastle-Ottawa Scale (NOS). An example of this scale is provided in online supplementary material 1A-D. In addition, publication bias will be assessed by visual inspection of a funnel plots and the Egger's and
Begg's regression tests. Summary estimates of the confidence placed on the evidence will be evaluated using the Grading of Recommendations Assessment Development and Evaluation (GRADE) of evidence about prognosis. GRADE for evidence about prognosis starts with highquality evidence that can then be rated down. These criteria are based on; (1) five domains diminishing confidence ( -1 for risk of bias, inconsistency, imprecision, indirectness and publication bias) and (2) two situations increasing confidence $(+1$ or +2 for large-very large effect size and $\mathrm{a}+1$ for a dose-response gradient (increasing pooled relative risks for fractures with increasing severity of AAC)). ${ }^{16}$

\section{Statistical analysis and data synthesis}

Analysis of outcome variables will be presented according to either: (1) AAC present or absent (2) AAC categorised as 'low' (referent-lowest reported group) versus 'high' (all other groups) or (3) dose-response when AAC was assessed in three or more groups. For the dose-response analysis, the lowest reported group (low AAC group) will be compared with the middle group(s) versus the highest reported AAC group (high AAC). Where data on more than three groups of AAC were presented the middle groups were combined as 'moderate AAC'. This approach was selected due to many studies reporting on variable number of AAC groups with the majority of studies using different cut-points for these groupings. Data on the severity of AAC quantification presented as a continuous measure or in three or more groupings of AAC will be used to determine the impact of increased abdominal aortic calcium load on outcomes. Where primary event data were reported in individual studies, pooled risk differences and risk ratios with $95 \%$ CIs will be calculated, from which, a summary estimate was determined using 
DerSimonian-Laird random effects models. For the AAC and BMD pooled analyses, estimates will be expressed as standardised mean difference with 95\% CI. Values will be considered significant if the $95 \%$ CI of the point estimate does not cross unity. Between-study heterogeneity will also be investigated by using subgroup analyses and the $\mathrm{I}^{2}$ statistic by study ID which quantifies inconsistency across studies to assess the impact of heterogeneity on the meta-analysis. ${ }^{1718}$ We will evaluate for heterogeneity using the $\mathrm{I}^{2}$ statistic and considered the $\mathrm{I}^{2}$ thresholds of $<25 \%, 25 \%-49 \%, 50 \%-75 \%$ and $>75 \%$ to represent low, moderate, high and very-high heterogeneity.

\section{Subgroup analysis and investigation of heterogeneity}

We will perform meta-regression of cohort characteristics to identify factors potentially explaining heterogeneity as well as performing subgroup analyses. $\mathrm{P}<0.01$ will be considered statistically significant for subgroup analyses. Preplanned subgroup analyses to explore statistical heterogeneity will include stratification by:

1. Subgroups based on clinical heterogeneity, for example, disease populations (general population, diabetics, chronic kidney disease, other) and age groups $(<60$ years, $60-69$ years and $\geq 70$ years).

2. Methodological heterogeneity, for example, AAC assessment methods (radiography, DXA or CT), thresholds to define high or severe AAC, fracture reporting and validation.

3. Statistical heterogeneity, for example, cohort characteristics (mean ages of the cohorts).

\section{Further analyses}

Where data on the severity of AAC quantification are presented as a continuous measure or in tertile/categories these data will be used to determine the impact of increased abdominal aortic calcium load on prognosis. Where AAC is not scored using the AAC24 scale equivalent values will be relative to estimated vertebral heights from similar aged populations. Where AAC is assessed by CT the categorical low vs moderate and high AAC will be used.

\section{Sensitivity analysis}

We will carry out sensitivity analyses for:

1. Large studies alone to establish how much they dominate the results $(n>500$ participants).

2. Methodology-we will assess the methodological quality of studies using the NOS for assessing the quality of nonrandomised studies in meta-analyses (online supplementary material 1). For the purpose of this sensitivity analysis, we will use three categories of quality (good, fair or poor).

3. Studies conducted in individuals without a history of a prior fracture (as this is the biggest risk factor for a new fracture).

4. Studies conducted in high-income versus low-income countries.
5. Studies that included non-osteoporotic fractures (fractures of the toes, fingers, face and skull fractures).

6. Study design bias comparing outcomes in cross-sectional and prospective studies (given that prospective studies may also include prevalent fractures and BMD measurements at baseline that can be analysed cross-sectionally).

\section{CONCLUDING STATEMENT}

Previous meta-analyses on this topic have a number of important limitations. By undertaking the preplanned comprehensive review and meta-analysis, we will gain better understanding of the relationship between AAC, BMD and increased fracture risk. The review will provide impetus for further research, diagnosis and treatment of this novel fracture risk factor. This review will also evaluate the quality of the published evidence and our confidence in the estimates for the meta-analysis, while identifying important knowledge gaps, potential sources of betweenstudy heterogeneity and issues with imaging, assessing or reporting of AAC in published studies.

\section{Ethics and dissemination}

The study will be submitted to a peer-reviewed journal and disseminated via research presentations.

\section{Author affiliations}

${ }^{1}$ Bone and Muscle Health Research Group, School of Clinical Sciences at Monash Health, Monash Medical Centre, Victoria, Australia

${ }^{2}$ The University of Sydney, Centre for Kidney Research, School of Public Health, Sydney Medical School, Children's Hospital at Westmead, New South Wales, Australia

${ }^{3}$ INSERM UMR 1033, University of Lyon, Hospices Civils de Lyon, Lyon, France ${ }^{4}$ School of Medical and Health Sciences, Edith Cowan University, Western Australia, Australia

${ }^{5}$ Medical School, Royal Perth Hospital Unit, The University of Western Australia, Western Australia, Australia

${ }^{6}$ Department of Renal Medicine, Sir Charles Gairdner Hospital, Western Australia, Australia

${ }^{7}$ Medical School, The University of Western Australia, Western Australia, Australia

${ }^{8}$ Division of Health Policy and Management, Park Nicollet Osteoporosis Center and Health Partners Institute, University of Minnesota, Minneapolis, USA

${ }^{9}$ Hinda and Arthur Marcus Institute for Aging Research, Hebrew Senior Life, Department of Medicine, Beth Israel Deaconess Medical Center, Harvard Medical School, Boston, Massachusetts, USA

${ }^{10}$ Department of Endocrinology and Diabetes, Sir Charles Gairdner Hospital, Perth, Australia

Contributors AR, KL, PS, DS, PE, MS, GW, WHL, JTS, DPK, RLP and JRL contributed to the study concept and design. AR and KL led the writing of the manuscript and is the primary designer of the protocol under the guidance of JRL, JTS and PS and all authors conceived the conceptual ideas presented in the revised protocol critically. All authors read and approved the revised version and final supported versions. JRL has the primary responsibility for the final content.

Funding The salaries of JRL and DS are supported by a National Health and Medical Research Council of Australia (NHMRC) Career Development Fellowship (ID: 1107474 and 1123014 , respectively).

Disclaimer Funding agencies had no input into any aspect of the design and management of this study.

Competing interests None declared.

Patient consent for publication Not required. 
Ethics approval The systematic review and meta-analysis does not require ethical approval.

Provenance and peer review Not commissioned; externally peer reviewed.

Open access This is an open access article distributed in accordance with the Creative Commons Attribution Non Commercial (CC BY-NC 4.0) license, which permits others to distribute, remix, adapt, build upon this work non-commercially, and license their derivative works on different terms, provided the original work is properly cited, appropriate credit is given, any changes made indicated, and the use is non-commercial. See: http://creativecommons.org/licenses/by-nc/4.0/.

\section{REFERENCES}

1. Farhat GN, Cauley JA. The link between osteoporosis and cardiovascular disease. Clin Cases Miner Bone Metab 2008;5:19-34.

2. Lillemark L, Ganz M, Barascuk N, et al. Growth patterns of abdominal atherosclerotic calcified deposits from lumbar lateral X-rays. Int J Cardiovasc Imaging 2010;26:751-61.

3. Kauppila LI, Mikkonen R, Mankinen P, et al. MR aortography and serum cholesterol levels in patients with long-term nonspecific lower back pain. Spine 2004;29:2147-52.

4. Thompson B, Towler DA. Arterial calcification and bone physiology: role of the bone-vascular axis. Nat Rev Endocrinol 2012;8:529-43.

5. Szulc P. Abdominal aortic calcification: a reappraisal of epidemiological and pathophysiological data. Bone 2016;84:25-37.

6. Touw WA, Ueland T, Bollerslev J, et al. Association of circulating wnt antagonists with severe abdominal aortic calcification in elderly women. J Endocr Soc 2017;1:26-38.

7. Warburton DE, Nicol CW, Gatto SN, et al. Cardiovascular disease and osteoporosis: balancing risk management. Vasc Health Risk Manag 2007;3:673-89.
8. Tankó LB, Christiansen C, Cox DA, et al. Relationship between osteoporosis and cardiovascular disease in postmenopausal women. J Bone Miner Res 2005;20:1912-20.

9. Ferrar L, Roux C, Felsenberg D, et al. Association between incident and baseline vertebral fractures in European women: vertebral fracture assessment in the Osteoporosis and Ultrasound Study (OPUS). Osteoporos Int 2012;23:59-65.

10. Black DM, Arden NK, Palermo L, et al. Prevalent vertebral deformities predict hip fractures and new vertebral deformities but not wrist fractures. Study of Osteoporotic Fractures Research Group. J Bone Min Res 1999;14:821-8.

11. Ross PD, Genant HK, Davis JW, et al. Predicting vertebral fracture incidence from prevalent fractures and bone density among nonblack, osteoporotic women. Osteoporos Int 1993;3:120-6.

12. Chen Z, Yu Y. Aortic calcification was associated with risk of fractures: a meta-analysis. J Back Musculoskelet Rehabil 2016;29:635-42.

13. Wei D, Zheng G, Gao Y, et al. Abdominal aortic calcification and the risk of bone fractures: a meta-analysis of prospective cohort studies. J Bone Miner Metab 2018;36:439-46.

14. Wang TK, Bolland MJ, van Pelt NC, et al. Relationships between vascular calcification, calcium metabolism, bone density, and fractures. J Bone Miner Res 2010;25:2777-85.

15. Stroup DF, Berlin JA, Morton SC, et al. Meta-analysis of observational studies in epidemiology: a proposal for reporting. Meta-analysis Of Observational Studies in Epidemiology (MOOSE) group. JAMA 2000;283:2008-12.

16. Iorio A, Spencer FA, Falavigna M, et al. Use of GRADE for assessment of evidence about prognosis: rating confidence in estimates of event rates in broad categories of patients. BMJ 2015;350:h870

17. Higgins JP, Thompson SG. Quantifying heterogeneity in a metaanalysis. Stat Med 2002;21:1539-58.

18. Higgins JP, Thompson SG, Deeks JJ, et al. Measuring inconsistency in meta-analyses. BMJ 2003;327:557-60. 